Received: $\quad 2019.08 .01$

Accepted: 2019.10.09

Published: $\quad 2019.12 .30$

\title{
Dexmedetomidine Regulates Proliferation, Apoptosis, Migration, and Invasion in Ovarian Cancer Cells via MiR-155-HIF-1 $\alpha$ Axis
}

\author{
ABE 1 Lihong Zheng \\ $B C D 2$ Ruimei Jia \\ DEF 3 Juan Zhao
}

1 Department of Anesthesiology, Harbin Medical University Cancer Hospital, Harbin, Heilongjiang, P.R. China

2 Department of Pain Clinic, The First Affiliated Hospital Harbin Medical University, Harbin, Heilongjiang, P.R. China

3 Department of Anesthesiology, Taixing People's Hospital, Taizhou, Jiangsu, P.R. China

Manuscript Preparation E

Literature Search F

Funds Collection G

Corresponding Author: Source of support:
Lihong Zheng, e-mail: zhenglihong53@yeah.net

Departmental sources

Background: Dexmedetomidine (DMED) is widely used as an adjuvant anesthetic, but how DMED regulates biological behavior of OC cells remains an area of active research. This study investigated the mechanism by which DMED regulates the proliferation, apoptosis, migration, and invasion abilities of OC cells.

Material/Methods: We determined the optimal concentration of DMED for use in treating SKOV3 cells. The biological activities of DMED-treated SKOV3 cells following transfection with miR-155 inhibitor or Si-HIF-1 $\alpha$ were measured by CCK- 8 assay, flow cytometry, wound healing assay, and Transwell assay. qRT-PCR and Western blot analysis were performed to assess the expression levels of apoptotic-related caspase-3 and Mcl-1. Luciferase reporter assay verified the targeting relationship of miR-155 and HIF-1 $\alpha$.

Result: $\quad$ miR-155 was downregulated while HIF-1 $\alpha$ was upregulated in SKOV3 cells. DMED dose-dependently reduced HIF- $1 \alpha$ expression in SKOV3 cells, and upregulated the expression of miR-155. DMED inhibited the proliferation, migration and invasion abilities of OC cells, but also contributed to apoptosis of SKOV3 cells, while transfection of miR-155 inhibitor inhibited the effect of DMED on SKOV3 cells. In contrast, transfection with si-HIF- $1 \alpha$ enhanced the effects of DMED on SKOV3 cells. HIF-1 $\alpha$ was found to be a target gene of miR-155.

Conclusions: Our results suggest that DMED blocks cell proliferation, migration, and invasion and accelerates cell apoptosis in OC.

MeSH Keywords:

Apoptosis • Cell Migration Assays • Cell Proliferation • Dexmedetomidine • Ovarian Neoplasms

Full-text PDF: https://www.medscimonit.com/abstract/index/idArt/919112

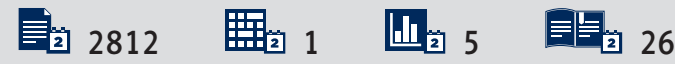




\section{Background}

Ovarian cancer $(O C)$, which is the third most common cause of gynecologic malignancies, has the highest mortality rate among gynecologic malignancies [1,2]. The absence of distinctive symptoms and lack of effective and available screening approaches in clinical practice makes early diagnosis of OC difficult. Unfortunately, stage of diagnosis is a major factor that determinates the survival rate of $\mathrm{OC}$ patients, and the average 5 -year survival rate for OC patients is only $44 \%[3,4]$. Although chemotherapy in advanced OC can achieve similar therapeutic efficiency as surgery, radical resection remains the primary treatment approach for OC $[5,6]$. Noticeably, anesthetic drugs must be properly used during $O C$ surgery to avoid adverse effects of anesthesia, such as respiratory depression and unstable vital signs [7]. Therefore, selection of anesthetic drugs essential for OC radical surgery.

Dexmedetomidine (DMED) is a selective $\alpha_{2}$-adrenoreceptor agonist that is a novel adjuvant analgesic-sparing agent used for reducing stress and protecting the cardiovascular system [8]. DMED can lead to local vasoconstriction, thus decreasing the systemic absorption and contributing to the safety and efficacy of intraperitoneal administration of local anesthetics [9]. Following cesarean section births, use of DMED can increase the analgesia effect from local anesthetics and can decrease adverse reactions [10]. In an OC rat model, DMED was found to inhibit activation of chemotherapy resistance pathways, thus improving therapeutic efficiency in patients with OC [11].

MicroRNAs (miRNAs) are mainly responsible for mediating the expression levels of many coding genes, consequently altering cellular pathways and thus affecting cell progression [12]. miR-155 can mediate DNA repair ability and sensitivity to ionizing radiation (IR) in triple-negative breast cancer [13]. Data also demonstrated that miR-155 can regulate cisplatin-induced apoptosis in $\mathrm{OC}$ by targeting $\mathrm{X}$-linked inhibitor of apoptosis protein (XIAP), suggesting the protective role of miR-155 in OC progression by promoting cell apoptosis [14]. However, whether miR-155 can regulate cell activity in DMED-treated OC cells remains to be determined. In this report, we tested the hypothesis that HIF- $1 \alpha$ is a target gene of miR-155. The miR-155-HIF- $1 \alpha-$ HO-1 signaling pathway was reported to involved in DMEDregulated endotoxin-induced acute lung injury [15]. However, whether DMED can regulate the miR-155-HIF-1 $\alpha$-HO-1 signal pathway to affect the viability of $O C$ cells remains an area of clinical uncertainty. In this regard, we hypothesize that DMED can regulate OC cell progression by the miR-155-HIF- $1 \alpha$ axis. Therefore, this work was undertaken to analyze the mechanism by which DMED regulates the proliferation, apoptosis, migration, and invasion abilities of SKOV3 cells via the miR-155-HIF-1 axis.

\section{Material and Methods}

\section{Cell culture}

OC cells SKOV 3 and ovarian epithelial cells HOSEpiC were purchased from American Type Culture Collection (ATCC, USA). Functionally, HOSEpiC cells were used as normal controls for OC cells. SKOV 3 cells were cultured in RPMI- 1640 culture medium (GIBCO, USA) containing 10\% FBS and 1\% double-antibody. The HOSEpiC cells were incubated in DMEM culture medium with $10 \% \mathrm{FBS}$ and $1 \%$ double-antibody. The incubation of SKOV3 and HOSEpiC cells was performed at $37^{\circ} \mathrm{C}$ with $5 \%$ $\mathrm{CO}_{2}$. DMED was purchased from Shanghai Lianshuo Biological Company. DMED of different concentrations $(0,1,10$, and $100 \mathrm{nM}$ ) were added to culture medium of SKOV3 cells. After 24-h incubation, culture medium was refreshed.

\section{Cell transfection}

SKOV3 cells were transfected with miR-155 inhibitor, miR-155 inhibitor negative control, si-HIF-1 $\alpha$, and its relative negative control (GenePharma, Shanghai). Lipofectamine 2000 transfection reagent (Invitrogen, USA) was used for transfection in accordance with its transfection instructions. Transfection experiments were conducted in triplicate.

\section{qRT-PCR}

Total RNAs were extracted using Trizol (Invitrogen, USA). The qualified RNAs were diluted to appropriate concentrations for reverse transcription using a reverse transcription kit (TaKaRa, Japan). All operations were performed based on kit protocols. Real-time quantitative RT-PCR was performed with a LightCycler 480 (Roche, Switzerland) fluorescence quantitative PCR instrument. The reaction conditions were conducted using a fluorescent quantitative RT-PCR kit (SYBR Green PCR kit, Dongsheng Biology). The detailed conditions for PCR were as follows: pre-denaturation at $95^{\circ} \mathrm{C}$ for $5 \mathrm{~min}$, denaturation at $95^{\circ} \mathrm{C}$ for $10 \mathrm{~s}$, annealing at $60^{\circ} \mathrm{C}$ for $10 \mathrm{~s}$, and extension at $72^{\circ} \mathrm{C}$ for $20 \mathrm{~s}$, for a total of 40 cycles. Three replicates were set up. U6 was selected as the internal reference of miRNA, and GAPDH served as the internal reference of mRNA. The $2^{-\Delta \Delta t}$ method was used to analyze data. The amplified primer sequences of each gene and its internal reference genes are detailed in Table 1.

\section{Western blot analysis}

Proteins were isolated and bathed in boiling water for $3 \mathrm{~min}$ for protein degeneration. The electrophoresis was conducted initially with $80 \mathrm{~V}$ for $30 \mathrm{~min}$, and then $120 \mathrm{~V}$ for $1 \sim 2 \mathrm{~h}$. The current of transfer membrane was $300 \mathrm{~mA}$ for $60 \mathrm{~min}$. After membrane transfer, the membrane was rinsed in washing solution for 1 2 $\mathrm{min}$, and then sealed with blocking buffer 
Table 1. Primer sequences used for RT-PCR.

\begin{tabular}{lc}
\hline Name of primer & \multicolumn{1}{c}{ Sequences } \\
\hline miR-155-F & GCAGGGTCCGAGGTATTC \\
\hline miR-155-R & GTGGGGATAGTGCTAATC \\
\hline U6-F & CTCGCTTCGGCAGCACATATACT \\
\hline U6-R & ACGCTTCACGAATTTGCGTGTC \\
\hline HIF-1 $\alpha-F$ & GTCTGTTGCTACAAGCCCCA \\
\hline HIF-1 $\alpha-R$ & TGGTAAGAAATTACGGGGGCA \\
\hline Caspase-3-F & GGGGGATATCGCTGTCATGG \\
\hline Caspase-3-R & CCGTACCAGAGCGAGATGAC \\
\hline MCl-1-F & GTTTTCAGCGACGGCGTAAC \\
\hline MCl-1-R & AAGGCAAACTTACCCAGCCT \\
\hline GAPDH-F & GTGGCTGGCTCAGAAAAAGG \\
\hline GAPDH-R & GGGGAGATTCAGTGTGGTG \\
\hline
\end{tabular}

F - forward; R - reverse; RT-PCR - reverse transcript polymerase chain reaction.

at room temperature for $60 \mathrm{~min}$, or incubated overnight at $4^{\circ} \mathrm{C}$. Primary antibodies of rabbit anti-human GAPDH (5174T), HIF-1 $\alpha$ (36169T), caspase-3 (9662S), Mcl-1(94296S), 1: 1000, Cell Signaling, Boston, USA) were added for incubation on a shaker at room temperature for $1 \mathrm{~h}$. After that, the membrane was washed 3 times for 10 min each time. Then, the membrane was incubated with secondary antibodies (HRP-marked goat anti-rabbit IgG, 1: 5000, Beijing Kangwei Century Biology Co., Beijing, China) at room temperature for $1 \mathrm{~h}$, followed by washing 3 times for 10 min each time. X-ray film was used for color pressing, developing, and fixing.

\section{Luciferase reporter gene experiment}

The online software TargetScan (http://www.targetscan.org/ mamm_31/) was used to predict the binding sites of miR-155 and HIF-1 $\alpha$. According to the predicted results, wild and mutation sequences of binding sites were designed and synthesized, respectively (wt-HIF-1 $\alpha$ and mut-HIF-1 $\alpha$ ). The wild sequence or mutation sequence on the binding site was inserted into the luciferase reporter gene vector (pGL3-Basic), and those 2 sequences were separately co-transfected with miR-155 mimic (30 nM, GenePharma) or miR-155 mimic negative control (30 nM, GenePharma) into HEK239T cells (Shanghai Sixin Biological Co.). After transfection, firefly luciferase activity and Renilla luciferase activity were detected in each group. Renilla luciferase activity was used as an internal reference, and the ratio of firefly luciferase activity to Renilla luciferase activity was regarded as the relative activity of luciferase. The experimental groups were named as follows: mimic NC+mut-HIF-1 $\alpha$ group, mimic NC+wtHIF-1 $\alpha$ group, mimic+mut-HIF-1 $\alpha$ group, and mimic + wt-HIF- $1 \alpha$ group. Luciferase intensity was measured 3 times.

\section{CCK-8 assay}

DMED-treated SKOV3 cells and suspensions of transfected SKOV3 cells of each group were seeded into 96-well plates (about 2000 cells in each well) with 3 replicates for per group. After incubation in an incubator for 24 h, 48 h, 72 h, and 96 h, $10 \mu \mathrm{l}$ of CCK-8 solution (Mosak Biology) was added to each well. Then, after incubation for another 1 4 h, the absorbance at $450 \mathrm{~nm}$ wavelength was measured.

\section{Flow cytometry}

The density of DMED-treated SKOV3 cells and suspensions of transfected SKOV3 cells of each group were adjusted to $10^{5} / \mathrm{ml}$. Then, $3 \mathrm{ml}$ of cell suspension was collected from each group into a $10-\mathrm{ml}$ centrifugal tube and centrifuged at $500 \mathrm{rpm}$ for $5 \mathrm{~min}$ before the culture medium was discarded. The suspension was washed with PBS and centrifuged at $500 \mathrm{rpm}$ for $5 \mathrm{~min}$. Then, the supernatant was discarded. Cells were re-suspended in $100 \mu \mathrm{l}$ of labeled solution and incubated for 10 min without light exposure. Annexin V-FITC $(5 \mu \mathrm{l})$ and PI $(5 \mu \mathrm{l})$ were added and gently mixed with cells for reaction for 10 min without light exposure. FITC fluorescence and PI fluorescence were measured using flow cytometry to analyze the apoptosis of SKOV3 cells. The migration ability of cells was measured 3 times.

\section{Scratch test}

DMED-treated SKOV3 cells and transfected SKOV3 cells in each group $\left(2 \times 10^{6}\right.$ per well) after transfection were inoculated into 6 -well plates. The cells in each group were inoculated into 3 wells and then incubated at $37^{\circ} \mathrm{C}$ with $5 \% \mathrm{CO}_{2}$ for $24 \mathrm{~h}$ until the cells formed a monolayer. Scratches were made on the monolayer cells with the tip of a pipette (sterile, $200 \mu \mathrm{l}$ ). Then, cells were washed with PBS before incubation at $37^{\circ} \mathrm{C}$ with $5 \% \mathrm{CO}_{2}$ for $24 \mathrm{~h}$. Scratch widths were observed and recorded under a microscope (200x) after culturing for $0 \mathrm{~h}$ and $24 \mathrm{~h}$. Migration rates of $\mathrm{OC}$ cells were analyzed based on scratch area. Migration rate $=(0 \mathrm{~h}$ scratch distance $-24 \mathrm{~h}$ scratch distance) $/ 0 \mathrm{~h}$ scratch distance.

\section{Transwell}

Matrigel matrix gel $(50 \mu \mathrm{g} / \mathrm{mL})$ was mixed with serum-free RPMI-1640 medium at a ratio of 1: 5 . Mixed matrix glue $(80 \mu \mathrm{L})$ was laid in a Transwell chamber with an $8-\mu \mathrm{m}$ aperture and placed on 24 -well plates at $37^{\circ} \mathrm{C}$ for $6 \mathrm{~h}$ to solidify the gel. OC cells in each group were collected after treatment for $48 \mathrm{~h}$. Serum-free RPMI-1640 medium was applied to adjust the cell concentration of each group to $2 \times 10^{5} / \mathrm{ml}$. We then added $200 \mu \mathrm{L}$ of cell suspension to the upper chamber and we added $600 \mu \mathrm{L}$ of RPMI-1640 medium with $1 \%$ low-serum to 


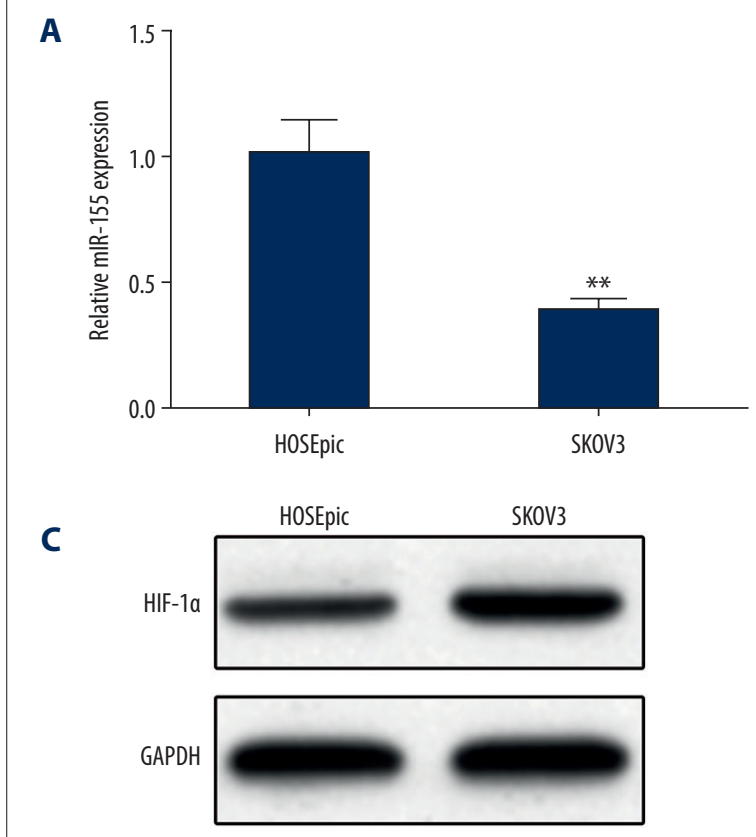

B
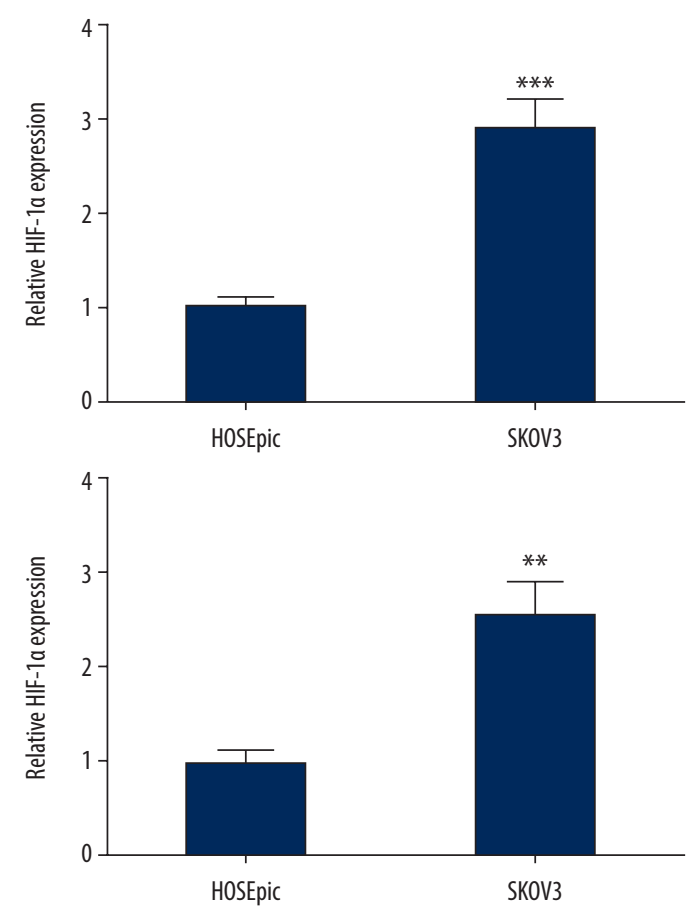

Figure 1. Decreased expression of miR-155 and elevated expression of HIF-1 $\alpha$ in SKOV3 cells. (A) The expression of miR-155 in SKOV 3 cells and HOSEpiC cells was determined by qRT-PCR. The expression of HIF- $1 \alpha$ in SKOV3 cells and HOSEpiC cells by (B) qRT-PCR and (C) Western blot; ${ }^{* *} P<0.01,{ }^{* * *} P<0.001$, compared to HOSEpiC group.

the lower chamber. After $24 \mathrm{~h}$ of incubation at $37^{\circ} \mathrm{C}$ and $5 \%$ $\mathrm{CO}_{2}$, the chamber was taken out. After PBS washing, methanol fixation and crystal violet staining, 5 visual fields were randomly selected under an inverted microscope to count cells. The number of cells passing through the Matrigel matrix gel was used to express the invasive ability of cells. Three multiple wells were set up in each experiment.

\section{Statistical analysis}

GraphPad software (version 7.0) was used for statistical analysis. All data are expressed as the mean value and standard deviation $(\bar{\chi} \pm s)$. We used the $t$ test to compare differences between 2 groups. One-way analysis of variance was used for comparison among groups. Dunnett's multiple comparisons test was performed for the post hoc comparison/test. $P<0.05$ was regarded as having statistical significance.

\section{Results}

\section{Decreased miR-155 and increased HIF-1 $\alpha$ in OC cells}

The expression levels of miR-155 and HIF- $1 \alpha$ in OC cells and HOSEpiC cells were measured by qRT-PCR and Western blot analysis, showing that miR-155 in SKOV3 cells was clearly lower than in HOSEpiC cells (Figure 1A), but the mRNA and protein expression levels of HIF-1 $\alpha$ in SKOV3 cell were obviously higher than in HOSEpiC cells (Figure 1B, 1C).

\section{DMED regulates miR-155 and HIF-1 $\alpha$ levels in OC cells}

SKOV3 cells subjected to DMED treatment (with concentrations of $0,1,10$, and $100 \mathrm{nM}$ ) had elevated expression of miR-155 (Figure 2A) and decreased expression of HIF-1 $\alpha$, and these differences were dose-dependent (Figure 2B, 2C). SKOV3 cells treated with $10 \mathrm{nM}$ of DMED had substantially upregulated miR-155 and notably downregulated expression of HIF- $1 \alpha$. Therefore, $10 \mathrm{nM}$ of DMED was selected for the following assays to explore the effects of DMED on OC biological behavior.

\section{DMED upregulates miR-155 to mediate proliferation, apoptosis, migration, and invasion of OC cells}

Transfection with miR-155 inhibitor reduced miR-155 expression in SKOV3 cells, but its expression was upregulated after DMED treatment. SKOV3 cells subjected to miR-155 inhibitor transfection and DMED treatment had substantially decreased miR-155 expression compared with cells treated with DMED alone (Figure 3A). After transfection of miR-155 inhibitor, the proliferation (Figure $3 \mathrm{~B}$ ), migration (Figure $3 \mathrm{~F}$ ), and invasion abilities (Figure 3G) of SKOV3 cells were enhanced, while 


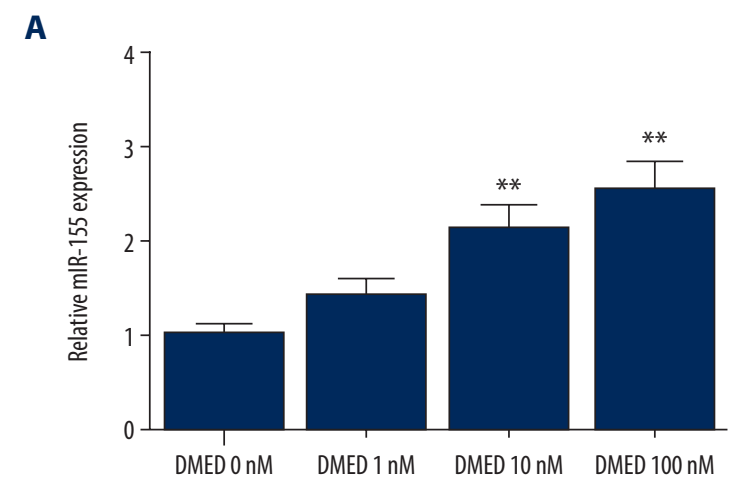

C

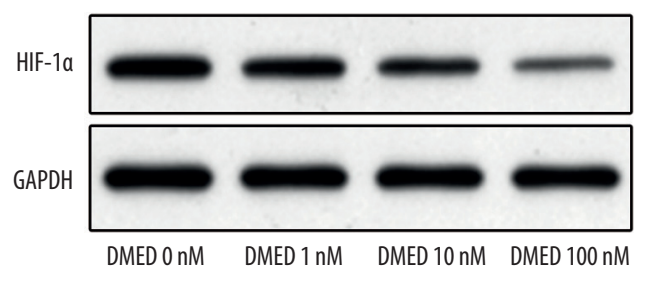

B
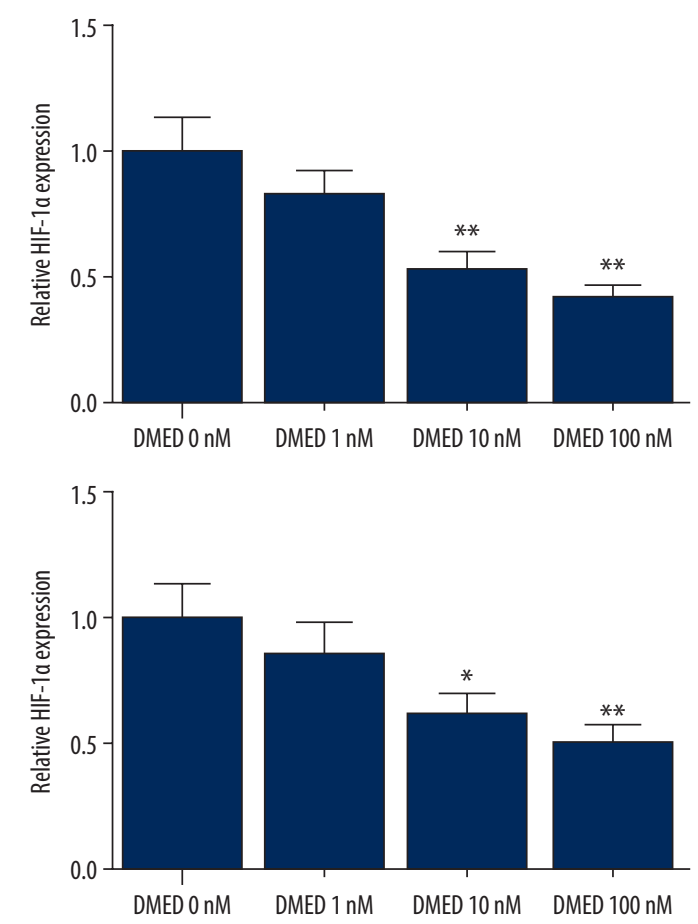

Figure 2. DMED upregulates miR-155 expression and downregulates HIF-1 $\alpha$ expression. (A) The expression of miR-155 in DMED (0, 1 , 10 and $100 \mathrm{nM}$ ) treated SKOV3 cells was detected by qRT-PCR; The mRNA and protein expression levels of HIF-1 $\alpha$ in SKOV3 cells by (B) qRT-PCR and (C) Western blot; ${ }^{*} P<0.05,{ }^{* *} P<0.01$, compared to DMED $0 \mathrm{nM}$ group; DMED - Dexmedetomidine.

cell apoptosis (Figure 3C) was clearly suppressed, as evidenced by increased expression level of $\mathrm{Mcl}-1$ and decreased expression level of caspase-3 (Figure 3D, 3E). However, the expression profile in DMED-treated SKOV3 cells was the opposite of cells transfected with miR-155 inhibitor. In cells subjected to DMED treatment and miR-155 inhibitor transfection, the effect of DMED on the proliferation, apoptosis, migration, and invasion of SKOV3 cells was offset by transfection of miR-155 inhibitor. These results demonstrate that DMED regulates $O C$ cell progression by upregulating miR-155 expression.

\section{HIF- $1 \alpha$ is a target gene of miR-155}

After transfection with si-HIF-1 $\alpha$, the expression of HIF- $1 \alpha$ in SKOV 3 cells was significantly decreased, while in SKOV3 cells transfected with miR-155 inhibitor, the expression of HIF-1 $\alpha$ was notably increased $(P<0.05)$ (Figure 4A, 4B). The binding site between miR-155 and HIF-1 $\alpha$ is represented in Figure $4 C$. There was no difference of luciferase activity between the mimic NC+mut-HIF- $1 \alpha$ group and mimic+mut-HIF-1 $\alpha$ group, whereas luciferase activity in the mimic + wt-HIF-1 $\alpha$ group was significantly reduced (mimic + wt-HIF- $1 \alpha$ group vs. mimic NC+wt-HIF-1 $\alpha$ group) (Figure 4D), suggesting that miR-155 can bind with HIF- $1 \alpha$.

\section{DMED regulates proliferation, apoptosis, migration, and invasion of OC cells via miR-155-HIF-1 $\alpha$ axis}

Transfection of si-HIF-1 $\alpha$ in DMED-treated SKOV3 cells can inhibit cell proliferation (Figure $5 \mathrm{~A}$ ), migration (Figure $5 \mathrm{E}$ ), and invasion (Figure 5F), and enhance cell apoptosis (Figure 5B), as shown by increased level of caspase- 3 expression and underexpression of $\mathrm{Mcl}-1$ (Figure 5C, 5D). These results suggest that HIF-1 deficiency further enhances the inhibitory effect of DMED on OC cells; therefore, DMED may inhibit OC progression through reducing HIF- $1 \alpha$ expression. The biological activity of DMED-treated SKOV3 cells co-transfected with si-HIF- $1 \alpha$ and miR-155 inhibitor showed a different expression pattern from that in DMED-treated SKOV3 cells transfected with si-HIF-1 $\alpha$, which suggests that miR-155 affects the biological behavior of OC cells via controlling and targeting HIF- $1 \alpha$, while DMED inhibits OC development via the miR-155-HIF-1 axis.

\section{Discussion}

To investigate the mechanism of DMED in the development of SKOV3 cells, the expression levels of miR-155 and HIF-1 $\alpha$ in ovarian epithelial cells HOSEpiC and DMED-treated OC cells were first examined. Then, we knocked down miR-155 and 


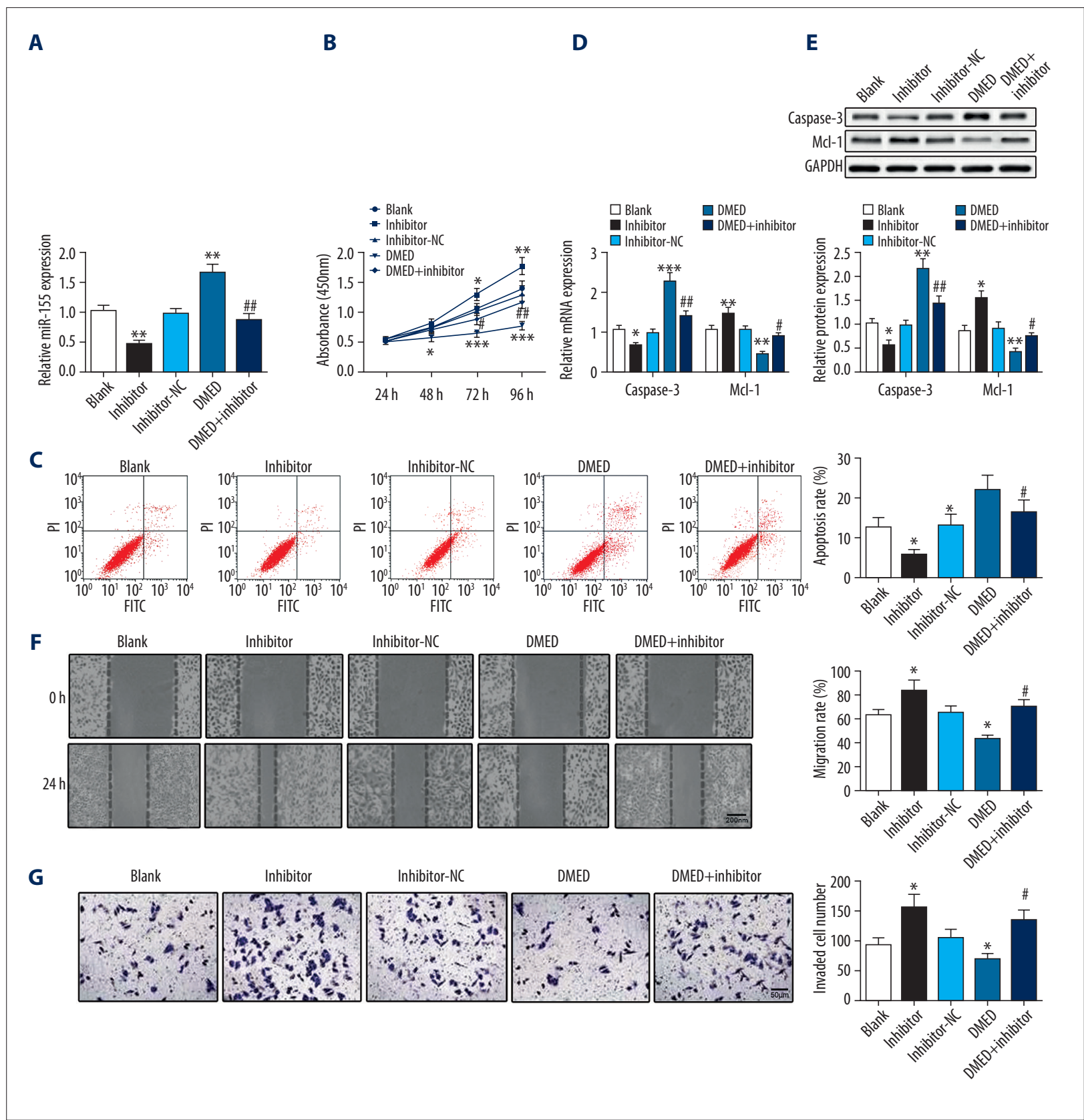

Figure 3. DMED upregulates miR-155 to mediate cell proliferation, apoptosis, migration, and invasion of OC cells. (A) qRT-PCR was performed to detect miR-155 expression in SKOV3 cells; (B) CCK-8 assay was used to measure SKOV3 cell proliferation; (C) Cell apoptosis rate was observed under the application of flow cytometry. The expression of caspase- 3 and Mcl-1 were determined by (D) qRT-PCR and (E) Western blot. Wound healing assay and Transwell were used to detect (F) cell migration and (G) Invasion ability. ${ }^{*} P<0.05,{ }^{* *} P<0.01,{ }^{* * *} P<0.001$, compared with Blank group; ${ }^{*} P<0.05,{ }^{\# \#} P<0.01$, compared with DMED group; DMED - Dexmedetomidine.

HIF- $1 \alpha$ expression levels to observe how miR-155 and HIF- $1 \alpha$ suppression can regulate $O C$ cell activity. Dual-luciferase reporter assay further identified the binding of miR-155 and HIF- $1 \alpha$. The results from this study support that DMED can inhibit the progression of OC cells by regulating the miR-155HIF-1 axis.
Although genetic factors and environmental factors are wellknown risk factors for OC [1], the detailed biological mechanism of OC occurrence still remains unknown. The biological function of $O C$ cells can be regulated by many miRNAs whose downregulation or overexpression has been detected in $\mathrm{OC}$ cells $[16,17]$. The first major finding in this study illustrated 


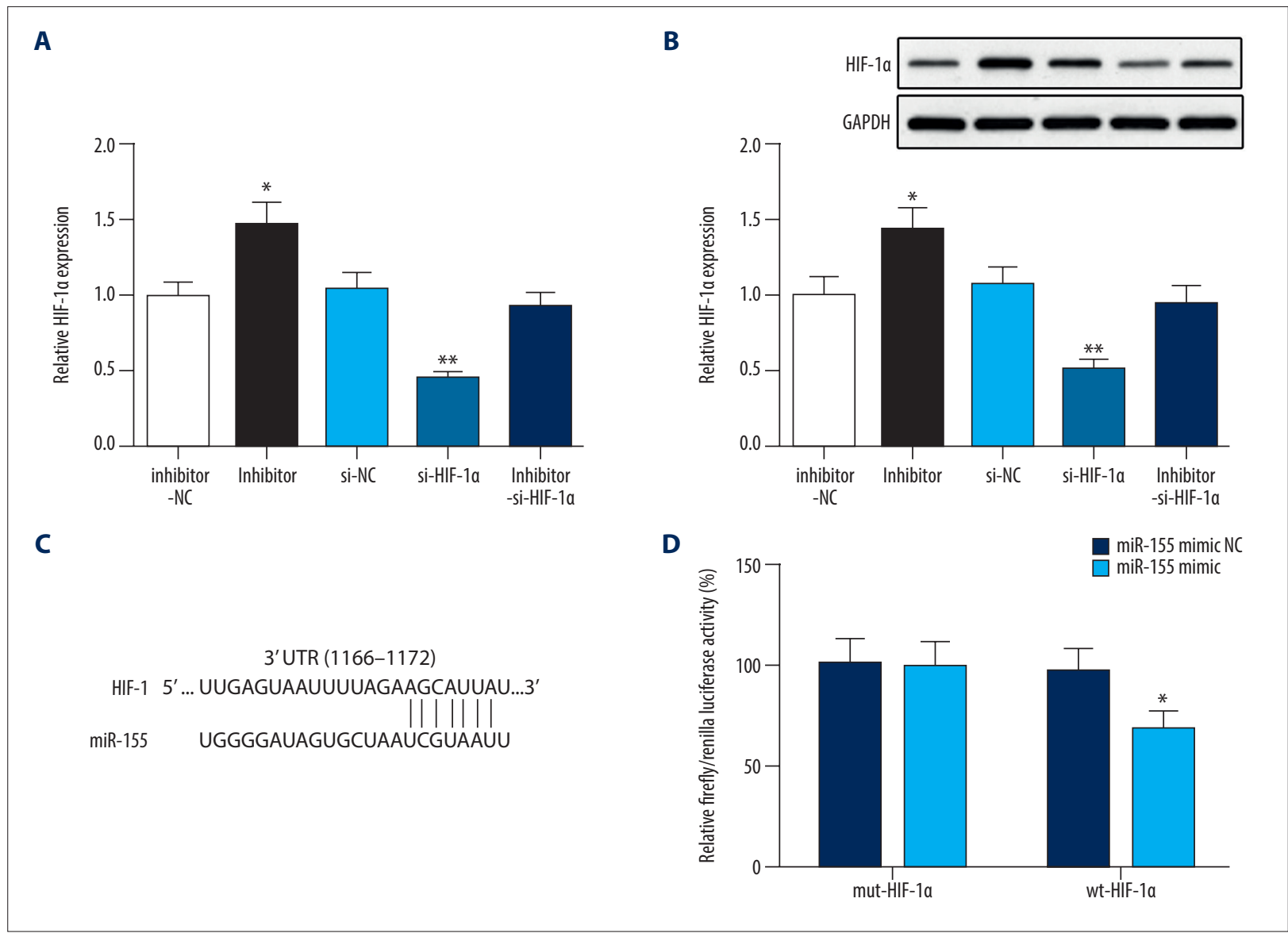

Figure 4. HIF- $1 \alpha$ was a target gene of miR-155. The expression of HIF- $1 \alpha$ in SKOV3 cells was measured by (A) qRT-PCR and (B) Western blot; (C) The binding site between miR-155 and HIF-1 $\alpha$ was on the 3'UTR; (D) The binding of miR-155 with HIF-1 $\alpha$ was verified by luciferase reporter gene assay; ${ }^{*} P<0.05,{ }^{* *} P<0.01$, compared inhibitor NC group or mimic NC+Wt-HIF- $1 \alpha$ group; NC - negative control.

that miR-155 expression in SKOV3 cells was notably decreased, whereas the expression of HIF-1 $\alpha$ behaved in the opposite fashion when compared with that in HOSEpiC cells. We speculate that the dysregulation of miR-155 and HIF-1 $\alpha$ might be involved in OC development. Further, the expression of miR-155 in DMED-treated cells was detected, supporting the regulatory role of DMED in miR-155 expression. Inflammation after surgery can promote cancer progression and metastasis, and DMED can decrease the risk of surgery-related tumor metastasis through inhibiting inflammation and exerting a sedative function [18]. In addition, administration of DMED can provide clinical benefits in oxygenation and lung mechanics for patients complicated with lung cancer and chronic obstructive pulmonary disease [19], but whether DMED provides similar benefit for OC patients remains to be explored. In this report, assessment of DMED-treated cell progression showed that DMED can hinder cell activity and potentiate cell apoptosis via the regulation of miR-155 expression. The role of miR-155 in tumors may vary in different contexts and it can either act as an oncomiR or an oncosuppressor miR [14]. Our study suggests that
miR-155 plays an important role as a tumor suppressor miR. Elevated serum expression of miR-155 was detected in osteosarcoma, and miR-155 has the potential to predict relapse of breast cancer [20,21]. Furthermore, accumulating evidence has emerged regarding the dysregulation of miR-155 in development of breast cancer drug resistance [22]. Consistent with our study, miR-155 was reported to regulate cell growth and proliferation of melanoma cells and to serve as a tumor suppressor [23]. Furthermore, miR-155 was long ago found to be down-regulated in OC cells and had antiproliferative and proapoptotic activity in OC by targeting claudin-1 [24]. However, the role of miR-155 in DMED-treated OC cells has been unclear.

This study also clarified that HIF-1 $\alpha$ is a target gene of miR-155. This result is in agreement with the conclusion in a prior study that found certain miRNAs, such as miR-155, can control HIF- $1 \alpha$ so as to form the HIF- $1 \alpha-$ miR- 155 pathway to maintain oxygen homeostasis [25]. Knockdown of HIF-1 $\alpha$ enhances cisplatin resistance in $\mathrm{OC}$ cells, and the pathway underlying HIF-1 $\alpha$-adjusted tumor metabolism could be a new target for 

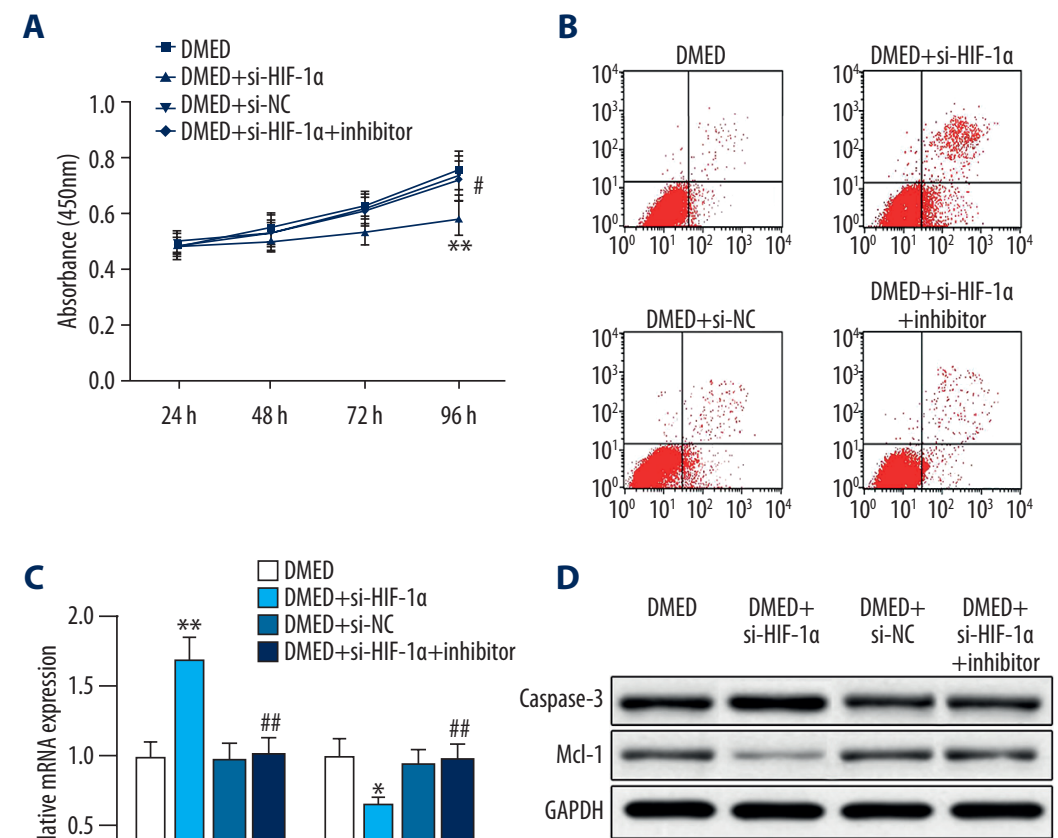

C

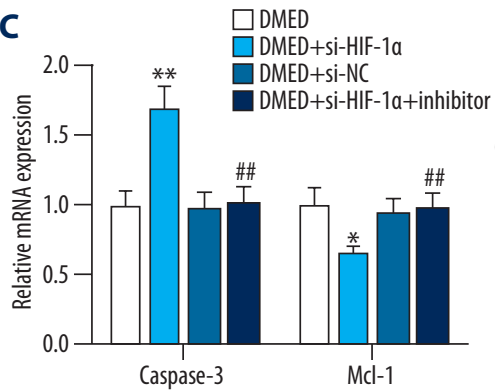

Caspase-3

Mcl-1
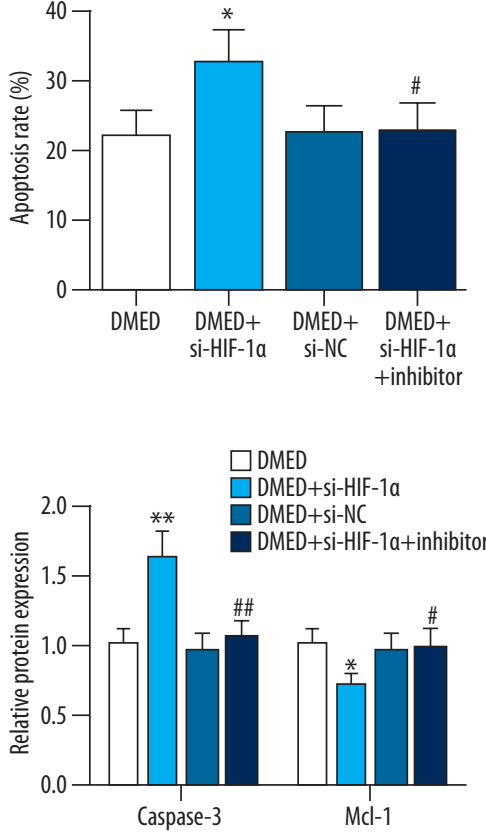

DMED+si-HIF-1a +inhibitor
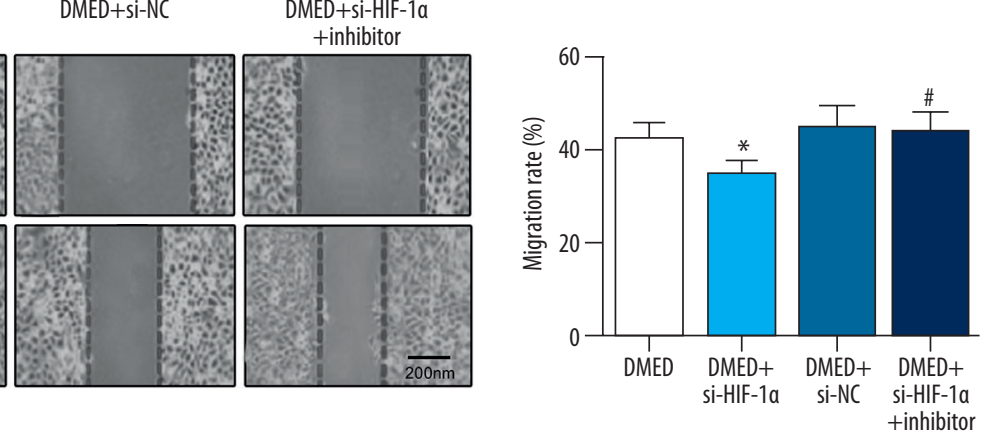

$\mathbf{F}$

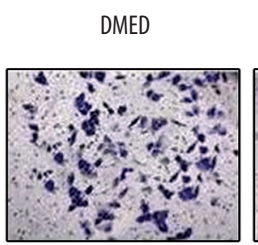

DMED+Si-HIF-1a
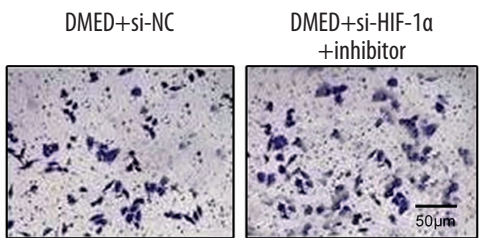

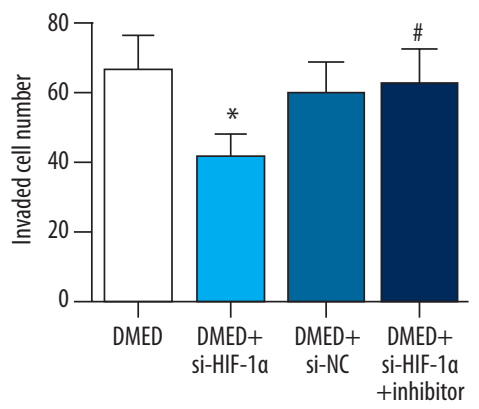

Figure 5. DMED regulates miR-155-HIF-1 $\alpha$ axis to mediate cell proliferation, apoptosis, migration, and invasion of OC cells. (A) Cell proliferation was inspected by CCK-8 assay; (B) Cell apoptosis was determined with flow cytometry; (C, D) The mRNA and protein expression levels of caspase- 3 and Mcl-1 were separately detected by qRT-PCR and Western blot; (E) Cell migration was detected by wound healing assay; (F) Cell invasion was determined using Transwell assay; ${ }^{*} P<0.05,{ }^{* *} P<0.01$, compared to DMED group; ${ }^{P} P<0.05, \# \#<0.01$, compared to DMED+si-HIF- $1 \alpha$ group; DMED - dexmedetomidine. 
overcoming cisplatin resistance in OC [26]. Our study further offers important evidence supporting our hypothesis that HIF- $1 \alpha$ is involved in OC. Therefore, we assessed the miR-155HIF- $1 \alpha$ pathway in therapy of OC. In the present work, transfection with miR-155 inhibitor inhibited the effects of DMED on the development of SKOV3 cells, while transfection with si-HIF- $1 \alpha$ promoted the effects of DMED on proliferation, apoptosis, migration, and invasion abilities of SKOV3 cells. These experiments further verified that DMED directly regulates miR-155 and HIF-1 $\alpha$ expression levels. It has become increasingly clear that DMED affects the progression of OC via the miR-155-HIF-1 $\alpha$ pathway.

\section{Conclusions}

Taken together, our research provides ample evidence that DMED affects the development of OC cells via the miR-155HIF- $1 \alpha$ pathway. Our results show the distinctive role of DMED in controlling $\mathrm{OC}$ progression and may have the possibility to be widely used for $\mathrm{OC}$ patients, possibly providing a new approach to treatment of OC. Considering the complicated biological mechanism and varied functions of DMED, further studies are required to better elucidate the roles of DMED in the biological behavior of OC cells.

\section{Conflict of interests}

None.

\section{References:}

1. Jelovac D, Armstrong DK: Recent progress in the diagnosis and treatment of ovarian cancer. Cancer J Clin, 2011; 61: 183-203

2. Salomon-Perzynski A, Salomon-Perzynska M, Michalski B et al: High-grade serous ovarian cancer: The clone wars. Arch Gynecol Obstet, 2017; 295 569-76

3. Gallotta V, Cicero C, Conte C et al: Robotic versus laparoscopic staging for early ovarian cancer: A case-matched control study. J Minim Invasive Gynecol, 2017; 24: 293-98

4. Ozga M, Aghajanian C, Myers-Virtue $S$ et al: A systematic review of ovarian cancer and fear of recurrence. Palliat Support Care, 2015; 13: 1771-80

5. Kehoe S, Hook J, Nankivell $M$ et al: Primary chemotherapy versus primary surgery for newly diagnosed advanced ovarian cancer (CHORUS): An openlabel, randomised, controlled, non-inferiority trial. Lancet, 2015; 386: 249-57

6. Narasimhulu DM, Khoury-Collado F, Chi DS: Radical surgery in ovarian cancer. Curr Oncol Rep, 2015; 17: 16

7. Dong $\mathrm{H}$, Zhang $\mathrm{Y}, \mathrm{Xi} \mathrm{H}$ : The effects of epidural anaesthesia and analgesia on natural killer cell cytotoxicity and cytokine response in patients with epithelial ovarian cancer undergoing radical resection. J Int Med Res, 2012; 40: $1822-29$

8. Xia M, Ji NN, Duan ML et al: Dexmedetomidine regulate the malignancy of breast cancer cells by activating alpha2-adrenoceptor/ERK signaling pathway. Eur Rev Med Pharmacol Sci, 2016; 20: 3500-6

9. Benito J, Monteiro B, Beaudry F et al: Efficacy and pharmacokinetics of bupivacaine with epinephrine or dexmedetomidine after intraperitoneal administration in cats undergoing ovariohysterectomy. Can J Vet Res, 2018; 82: $124-30$

10. Mo Y, Qiu S: Effects of dexmedetomidine in reducing post-cesarean adverse reactions. Exp Ther Med, 2017; 14: 2036-39

11. Cai $\mathrm{QH}$, Tang Y, Fan SH et al: In vivo effects of dexmedetomidine on immune function and tumor growth in rats with ovarian cancer through inhibiting the p38MAPK/NF-kappaB signaling pathway. Biomed Pharmacother, 2017; 95: $1830-37$

12. Park GB, Kim D: MicroRNA-503-5p inhibits the CD97-mediated JAK2/STAT3 pathway in metastatic or paclitaxel-resistant ovarian cancer cells. Neoplasia, 2019; 21: 206-15

13. Gasparini P, Lovat F, Fassan $M$ et al: Protective role of miR-155 in breast cancer through RAD51 targeting impairs homologous recombination after irradiation. Proc Natl Acad Sci USA, 2014; 111: 4536-41
14. Chen W, Huang L, Hao C et al: MicroRNA-155 promotes apoptosis in SKOV3, A2780, and primary cultured ovarian cancer cells. Tumour Biol, 2016; 37: 9289-99

15. Huang T, Gao Y, Luo K et al: [Effect of DMED on microRNA-155-hypoxia-inducible factor-1 $\alpha$-heme oxygenase- 1 signaling pathway in a rat model of endotoxin-induced acute lung injury.] Chinese Journal of Anesthesiology, 2016; 36: 214-18 [in Chinese]

16. Dahiya N, Morin PJ: MicroRNAs in ovarian carcinomas. Endocr Relat Cancer, 2010; 17: F77-89

17. Zhang S, Lu Z, Unruh AK et al: Clinically relevant microRNAs in ovarian cancer. Mol Cancer Res, 2015; 13: 393-401

18. Cata JP, Singh V, Lee BM et al: Intraoperative use of dexmedetomidine is associated with decreased overall survival after lung cancer surgery. Anaesthesiol Clin Pharmacol, 2017; 33: 317-23

19. Lee $\mathrm{SH}$, Kim N, Lee CY et al: Effects of dexmedetomidine on oxygenation and lung mechanics in patients with moderate chronic obstructive pulmonary disease undergoing lung cancer surgery: A randomised double-blinded trial. Eur J Anaesthesiol, 2016; 33: 275-82

20. Basova P, Pesta M, Sochor M et al: Prediction potential of serum miR-155 and miR-24 for relapsing early breast cancer. Int J Mol Sci. 2017; 18: pii: E2116

21. Bhattacharya S, Chalk AM, Ng AJ et al: Increased miR-155-5p and reduced miR-148a-3p contribute to the suppression of osteosarcoma cell death. Oncogene, 2016; 35: 5282-94

22. Yu DD, Lv MM, Chen WX et al: Role of miR-155 in drug resistance of breast cancer. Tumour Biol, 2015; 36: 1395-401

23. DiSano JA, Huffnagle I, Gowda R et al: Loss of miR-155 upregulates WEE1 in metastatic melanoma. Melanoma Res, 2019; 29: 216-19

24. Qin W, Ren Q, Liu T et al: MicroRNA-155 is a novel suppressor of ovarian cancer-initiating cells that targets CLDN1. FEBS Lett, 2013; 587: 1434-39

25. Yang D, Wang J, Xiao $M$ et al: Role of mir-155 in controlling HIF-1alpha level and promoting endothelial cell maturation. Sci Rep, 2016; 6: 35316

26. Ai Z, Lu Y, Qiu S et al: Overcoming cisplatin resistance of ovarian cance cells by targeting HIF-1-regulated cancer metabolism. Cancer Lett, 2016; 373: 36-44 